CTP-TAMU-43/97

LPTENS-97/50

hep-th/9710243

Oct. 1997

\title{
Fibre Bundles and Generalised Dimensional Reductions
}

\author{
I.V. Lavrinenko ${ }^{\dagger}$, H. Lü ${ }^{\ddagger}$ and C.N. Pope ${ }^{\dagger 1}$ \\ ${ }^{\dagger}$ Center for Theoretical Physics, Texas A $₫ M$ University, College Station, Texas 77843 \\ ${ }^{\ddagger}$ Laboratoire de Physique Théorique de l'École Normale Supérieure ${ }^{2}$ \\ 24 Rue Lhomond - 75231 Paris CEDEX 05
}

\begin{abstract}
$\underline{\text { ABSTRACT }}$
We study some geometrical and topological aspects of the generalised dimensional reduction of supergravities in $D=11$ and $D=10$ dimensions, which give rise to massive theories in lower dimensions. In these reductions, a global symmetry is used in order to allow some of the fields to have a non-trivial dependence on the compactifying coordinates. Global consistency in the internal space imposes topological restrictions on the parameters of the compactification as well as the structure of the space itself. Examples that we consider include the generalised reduction of the type IIA and type IIB theories on a circle, and also the massive ten-dimensional theory obtained by the generalised reduction of $D=11$ supergravity.
\end{abstract}

\footnotetext{
${ }^{1}$ Research supported in part by DOE Grant DE-FG03-95ER40917

${ }^{3}$ Unité Propre du Centre National de la Recherche Scientifique, associée à l'École Normale Supérieure et à l'Université de Paris-Sud
} 


\section{Introduction}

The standard Kaluza-Klein procedure for dimensional reduction on a circle or a torus consists of two elements. Firstly, one performs a Fourier expansion of all the higher-dimensional fields, in terms of the mode functions on the circle or torus. Then, one usually follows this by a truncation in which all but the zero mode sectors are discarded. The two steps can equivalently be performed simultaneously, by taking the lower-dimensional fields to be independent of the compactification coordinates. This procedure is guaranteed to be consistent, i.e. all solutions of the lower-dimensional theory will also be solutions of the original higherdimensional one. This can be seen by performing the reduction and truncation at the level of the equations of motion of the higher-dimensional theory. In general, the consistency of a truncation is threatened if non-linear terms involving the fields that are retained can generate mode-function dependences of fields that are being truncated to zero. Clearly this cannot happen here, since one cannot generate non-zero Fourier modes from products of zero modes. Thus the truncation is consistent.

What is less familiar is that in circle or torus compactifications there can be slightly more general truncations that are also consistent, even when certain dependences on the compactification coordinates are allowed. Suppose, for example, we consider a reduction on a single circle with coordinate $z$. If a particular field $\Phi$ appears in the higher-dimensional equations of motion only via its derivative, then it follows that the usual Kaluza-Klein truncation $\hat{\Phi}(x, z)=\Phi(x)$ can be generalised to $\hat{\Phi}(x, z)=\Phi(x)+m z$, where $m$ is an arbitrary constant. There will still be no $z$ dependence in the higher-dimensional equations of motion, and consequently the truncation to the lower-dimensional theory will still be consistent. A typical simple situation where such a generalised reduction can be performed is when the field $\Phi$ is an axion.

In fact the above example is still not the most general kind of reduction that one may consider. The essential feature in that example was that the field $\Phi$ was subject to the shift symmetry $\Phi \rightarrow \Phi+c$, where $\mathrm{c}$ is a constant. This is a global $\mathbb{R}$ symmetry. One could still, for example, perform a generalised reduction even in a different choice of field variables, related to the original ones by local transformations, in which the field $\Phi$ was no longer covered everywhere by a derivative. All that is necessary is that the theory admit the global $\mathbb{R}$ symmetry. The generalised reduction could then be effected by performing a global symmetry transformation with a parameter $c$ that is replaced by the quantity $m z$, and then following this with a "standard" ( $z$-independent) Kaluza-Klein reduction. The symmetry under constant shifts $c$ would then ensure that after setting $c=m z$, only terms where 
a derivative falls on $z$ would survive, and thus the transformed reduction scheme would still give $z$ independence, hence ensuring the consistency of the truncation. In general, the resulting lower-dimensional theory will be a massive theory, with masses proportional to the parameter $m$ [1, 2, 3, 田, 年, 6].

Further generalisations of the above scheme are clearly possible, in which one considers any global symmetry of the higher-dimensional equations of motion, and then replaces the constant parameters of the transformations by terms linearly dependent on the compactification coordinates on the torus or circle. For example, we could consider the $S L(2, \mathbb{R})$ global symmetry of the type IIB theory, and perform a generalised reduction on a circle, by first performing an $S L(2, \mathbb{R})$ transformation in which the three parameters are allowed to be linearly dependent on the compactification coordinate. Of course there will really only be two, rather than three, independent mass parameters in the nine-dimensional theory, since transformations in the stability subgroup of $S L(2, \mathbb{R})$ will not have any effect. Another example of this kind is a generalised circle reduction of the type IIA theory. This theory has a global $\mathbb{R}$ symmetry of the action, corresponding, in the usual field variables, to a shift of the dilaton accompanied by appropriate rescalings of the other fields. (One can, in this case, redefine fields to absorb all the non-derivative dilaton dependence, but, as we have remarked above, it is not essential to do this in order to be able to perform the generalised reduction.)

There are also global scaling symmetries in supergravities that lie outside the CremmerJulia symmetries [7, 8] that are usually considered (see also [9]). Specifically, there is a constant scaling symmetry that leaves the equations of motion invariant, although it is not a symmetry of the action since it scales it uniformly by an overall constant factor. (In fact this feature, of leaving only the equations of motion invariant, arises also for the CremmerJulia symmetries in even dimensions.) The scaling symmetry is the generalisation of the scaling symmetry of the pure Einstein theory under $g_{\mu \nu} \rightarrow \lambda^{2} g_{\mu \nu}$, where $\lambda$ is a constant. For example, in eleven-dimensional supergravity the equations of motion are invariant under constant field scalings which, in the bosonic sector, take the form

$$
g_{\mu \nu} \longrightarrow \lambda^{2} g_{\mu \nu}, \quad A_{\mu \nu \rho} \longrightarrow \lambda^{3} A_{\mu \nu \rho}
$$

This global $\mathbb{R}$ symmetry too can be used to allow generalised Kaluza-Klein reductions.

\footnotetext{
${ }^{1}$ The significance of this scaling symmetry for understanding the lower-dimensional Cremmer-Julia symmetries was recently observed in 10. It was also used in 11] to construct spectrum-generating symmetries for the BPS solitons, and in the rheonomy approach to supersymmetry; see, for example 12 .
} 
In this paper, we shall explore a number of aspects of the generalised reduction of supergravity theories. We begin in section 2 by considering some simple examples involving generalised reductions for axionic scalars. Our discussion also addresses some geometrical and topological aspects of the internal compactifying spaces. Although we shall be restricting attention to compactifications on circles and products of circles, it can nevertheless be that topologically non-trivial "twists" can arise, due to the imposition of the generalised reduction ansatz. Thus, as we shall show, the total internal compactifying space can sometimes have the structure of a non-trivial torus bundle over a base torus. In particular, we show how, in the cases where the internal space is a torus bundle, there are topologicallyimposed relations between the compactification periods of the different circles. These in turn imply relations between the various mass parameters.

In section 3 we present a general discussion of more general reduction procedures, in which fields other than axions are allowed non-trivial dependence on the compactifying coordinates. In section 4, we give some examples of this kind, for theories with global symmetries that include shifting symmetries of the dilaton. Our examples here include the generalised reduction of type IIA to $D=9$, using the dilatonic $\mathbb{R}$ symmetry, and generalised reductions using the global $S L(2, \mathbb{R})$ symmetries of the type IIB theory in $D=10$ and the type IIA theory in $D=9$.

In section 4, we consider the generalised reduction of eleven-dimensional supergravity to $D=10$, using the global scaling $\mathbb{R}$ symmetry. This gives rise to a massive type IIA supergravity in $D=10$. In fact this theory was also considered recently in [13], where it was obtained by different, although not entirely unrelated, techniques. It is, unfortunately, not the same as the massive IIA theory found by Romans [14, which has found a rôle in recent times in connection with D8-branes in string theory [15]. Nonetheless, as we shall show, the new theory has its own interesting features, including the fact that it admits a ten-dimensional de Sitter spacetime solution.

The paper ends with concluding remarks in section 6. Some details of Kaluza-Klein reductions are included in an appendix.

\section{Domain walls and torus bundles}

\subsection{Generalised reductions with axionic scalars}

In this section, we shall consider domain walls that arise as solutions of the massive theories obtained by the generalised Kaluza-Klein reduction of eleven-dimensional supergravity. We 
shall concentrate on cases where the massive theories are obtained by generalised toroidal reductions, The simplest example is provided by the Scherk-Schwarz reduction of maximal supergravity in nine dimensions, giving a massive theory in $D=8$. The nine-dimensional theory has one axion, namely $\mathcal{A}_{0}^{(12)}$, which can be reduced to eight dimensions using the generalised Kaluza-Klein ansatz

$$
\mathcal{A}_{0}^{(12)}\left(x, z_{3}\right)=m z_{3}+\mathcal{A}_{0}^{(12)}(x)
$$

One can easily see from the structure of the Chern-Simons modifications to the field strengths in $D=9$, given in (A.5), that after suitable field redefinitions this axion can be covered by a derivative everywhere, and thus the reduction to $D=8$ will be consistent since no $z_{3}$ dependence will occur in the nine-dimensional equations of motion. This means that one can consistently extract eight-dimensional equations of motion, whose solutions will all be solutions of the nine-dimensional theory, and hence also of the eleven-dimensional theory. The resulting eight-dimensional theory is maximally supersymmetric, with mass terms for certain of the gauge potentials, and in addition a cosmological term of the form [2]

$$
\mathcal{L}_{\text {cosmo }}=-\frac{1}{2} e m^{2} e^{\vec{b}_{123} \cdot \vec{\phi}}
$$

where $\vec{b}_{123}$ is a constant 3 -vector characterising the couplings of the three dilatonic scalars $\vec{\phi}=\left(\phi_{1}, \phi_{2}, \phi_{3}\right)$ coming from the diagonal components of the compactifying metric [16, 2].

One solution of the massive theory that is of particular interest is a domain wall, which is effectively like the ground state of the massive eight-dimensional theory. (The massive theory admits no maximally-symmetric ground state, i.e. neither Minkowski spacetime nor an anti de Sitter spacetime.) The domain-wall solution is given by

$$
\begin{aligned}
d s_{8}^{2} & =H^{1 / 6} d x^{\mu} d x_{\mu}+H^{7 / 6} d y^{2}, \\
e^{\phi} & =H^{-a / 2}, \quad H=1+m|y|,
\end{aligned}
$$

where $a \phi=\vec{b}_{123} \cdot \vec{\phi}$ and $a=\sqrt{19 / 3}$. This is a BPS-saturated solution, which preserves $\frac{1}{2}$ of the supersymmetry. It can be viewed as the generalisation of the usual BPS-saturated $p$-brane solitons to the case where the field strength supporting the solution is a 0 -form, namely the constant $m$, carrying a magnetic charge.

When oxidised back to $D=11$, the metric becomes

$$
d s_{11}^{2}=d x^{\mu} d x_{\mu}+H d y^{2}+H\left(d z_{2}^{2}+d z_{3}^{2}\right)+H^{-1}\left(d z_{1}+m z_{3} d z_{2}\right)^{2}
$$

The three-dimensional manifold on which the eleven-dimensional theory is compactified is described by the coordinates $\left(z_{1}, z_{2}, z_{3}\right)$. It has the structure of a $U(1)$ principal fibre bundle, 
with coordinate $z_{1}$, over a two-dimensional base torus with coordinates $\left(z_{2}, z_{3}\right)$. The bundle is non-trivial, since there is a connection form $\mathcal{A}_{1}^{(1)}=m z_{3} d z_{2}$ on the fibre, which can be seen to be non-trivial since its curvature $\mathcal{F}_{2}^{(1)}=d \mathcal{A}_{1}^{(1)}=m d z_{2} \wedge d z_{3}$ has a non-vanishing integral over the volume of the 2-torus. This imposes a periodicity condition on $z_{1}$, which we shall discuss in more detail later.

At a fixed value of the transverse-space coordinate $y$, the internal metric in (2.4) is of the form

$$
d s^{2}=\lambda^{2}\left(d z_{2}^{2}+d z_{3}^{2}\right)+\lambda^{-2}\left(d z_{1}+m z_{3} d z_{2}\right)^{2}
$$

where $\lambda$ is a constant. This metric is homogeneous, as can easily be seen from the fact that the vielbeins $e^{1}=\lambda^{-1}\left(d z_{1}+m z_{3} d z_{2}\right), e^{2}=\lambda d z_{2}, e^{3}=\lambda d z_{3}$ satisfy

$$
d e^{1}=-m \lambda^{-3} e^{2} \wedge e^{3}, \quad d e^{2}=0, \quad d e^{3}=0
$$

and thus the structural coefficients $C_{b c}{ }^{a}$ in the exterior derivatives $d e^{a}=-\frac{1}{2} C_{b c}{ }^{a} e^{b} \wedge e^{c}$ are constants. The spin connection and curvature 2 -form are given by

$$
\begin{aligned}
& \omega_{12}=-\frac{1}{2} m \lambda^{-3} e^{3}, \quad \omega_{23}=\frac{1}{2} m \lambda^{-3} e^{1}, \quad \omega_{31}=-\frac{1}{2} m \lambda^{-3} e^{2}, \\
& \Theta_{12}=\frac{1}{4} m^{2} \lambda^{-6} e^{1} \wedge e^{2}, \quad \Theta_{23}=-\frac{3}{4} m^{2} \lambda^{-6} e^{2} \wedge e^{3}, \quad \Theta_{31}=\frac{1}{4} m^{2} \lambda^{-6} e^{3} \wedge e^{1} .
\end{aligned}
$$

In fact, the metric (2.5) is precisely of the form of the Bianchi II metrics in the standard classification of three-dimensional homogeneous spaces [17]. All solutions of the eightdimensional massive theory will preserve the topological structure of this three-dimensional internal space.

Many further examples of massive supergravities coming from M-theory can be found in all dimensions $D \leq 8$ [3, 4, 6, 6]. First of all, one can perform a Scherk-Schwarz reduction on any one of the axions that arises in any of the usual maximal supergravities in $D \leq 9$. A natural generalisation is then to consider cases where $N \geq 2$ axions are simultaneously subjected to the Scherk-Schwarz reduction procedure. In order to be able to do this, it is necessary that they can be simultaneously covered everywhere by derivatives. In other words, there should be at least an $R^{N}$ symmetry describing the simultaneous global shift symmetries of the $N$ axions. However, it is not guaranteed that every such massive theory will admit a domain wall solution. The situation is analogous to that for multi-charge $p$ brane solutions using field strengths of degree $\geq 1$, where only certain combinations of field strengths may be used in their construction [16, 18]. In both the present case where the field strengths have degree $n=0$, and the more usual cases with field strengths of degree $n \geq 1$, the criterion for the existence of multi-charge solutions is the same, namely that they 
exist if the set of $N$ participating fields strengths have dilaton vectors $\vec{c}_{\alpha}(\alpha=1, \ldots, N)$ that satisfy 16

$$
\vec{c}_{\alpha} \cdot \vec{c}_{\beta}=4 \delta_{\alpha \beta}-\frac{2(n-1)(D-n-1)}{D-2} .
$$

In the present context, every set of cosmological terms whose dilaton vectors satisfy [6]

$$
\vec{c}_{\alpha} \cdot \vec{c}_{\beta}=4 \delta_{\alpha \beta}+\frac{2(D-1)}{D-2}
$$

can be arrived at by an appropriate Scherk-Schwarz reduction on multiple axions. Other cases where simultaneous Scherk-Schwarz reductions are possible, but the resulting dilaton vectors for the cosmological terms do not satisfy (2.9), correspond to massive theories that do not admit any domain wall solutions (nor do they admit Minkowski or anti de-Sitter solutions).

An example of a massive theory involving simultaneous Scherk-Schwarz reductions was presented in [2], where it was shown that three of the four axions in the massless eightdimensional theory, for example $\mathcal{A}_{0}^{(12)}, \mathcal{A}_{0}^{(13)}$ and $A_{0}^{(123)}$, can be simultaneously covered by derivatives. Using this $R^{3}$ symmetry, a massive supergravity in $D=7$ was constructed, whose "cosmological terms" are of the form

$$
\mathcal{L}_{\text {cosmo }}=-\frac{1}{2} m_{1}^{2} e^{\vec{b}_{124} \cdot \vec{\phi}}-\frac{1}{2} m_{3}^{2} e^{\vec{a}_{1234} \cdot \vec{\phi}}-\frac{1}{2}\left(m_{2}-m_{1} \mathcal{A}_{0}^{(23)}\right)^{2} e^{\vec{b}_{134} \cdot \vec{\phi}}
$$

If $m_{1}$ is non-zero, the redefinition $\mathcal{A}_{0}^{(23)} \rightarrow \mathcal{A}_{0}^{(23)}+m_{2} / m_{1}$ turns the final term into a mass term for $\mathcal{A}_{0}^{(23)}$. Thus whether or not $m_{1}$ is non-zero, the theory actually has just two cosmological terms, and admits 2-charge domain wall solutions using $e^{\vec{a}_{1234} \cdot \vec{\phi}}$ and either $e^{\vec{b}_{124} \cdot \vec{\phi}}$ or $e^{\vec{b}_{134} \cdot \vec{\phi}}$. It is easily verified from the equations in the appendix that in both cases (2.9) is satisfied, and therefore there exist domain-wall solutions carrying two charges, namely $m_{1}$ and $m_{3}$, or $m_{2}$ and $m_{3}$. Configurations in the seven-dimensional theory are reinterpreted from the eleven-dimensional point of view as $U(1)$ bundles over $T^{3}$. This can be easily seen from the structure of the vielbeins in the four internal directions, which take the form

$$
\begin{aligned}
e^{1} & \sim\left(d z_{1}+m_{1} z_{4} d z_{2}+m_{2} z_{4} d z_{3}+\mathcal{A}_{1}^{(1)}+\mathcal{A}_{0}^{(1 j)} d z_{j}\right) \quad j>1, \\
e^{i} & \sim\left(d z_{i}+\mathcal{A}_{1}^{(i)}+\mathcal{A}_{0}^{(i j)} d z_{j}\right) \quad j>i>1
\end{aligned}
$$

where we have omitted the exponential dilatonic prefactors. In the domain wall solutions themselves, only one charge appears in the vielbeins (the other is carried by the 4-form field strength). This is manifest if $m_{1}=0$; if on the other hand $m_{1} \neq 0$ we will have $\mathcal{A}_{0}^{(23)}=m_{2} / m_{1}$, and the charge is associated with the single potential $z_{4}\left(m_{1} d z_{2}+m_{2} d z_{3}\right)$. 
More complicated examples of massive supergravities can be obtained where the fibres of the internal space are higher-dimensional tori $T^{n}$ with $n>1$. For example, there is a seven-dimensional massive theory obtained by subjecting $\mathcal{A}_{0}^{(13)}$ and $\mathcal{A}_{0}^{(23)}$ to Scherk-Schwarz reductions from $D=8$. From the eleven-dimensional point of view, configurations in $D=7$ are then interpreted in terms of $T^{2}$ bundles over $T^{2}$, as can be seen from the form of the internal vielbeins

$$
\begin{aligned}
& e^{1} \sim\left(d z_{1}+m_{1} z_{4} d z_{3}+\cdots\right), \quad e^{2} \sim\left(d z_{2}+m_{2} z_{4} d z_{3}+\cdots\right), \\
& e^{3} \sim\left(d z_{3}+\cdots\right), \quad e^{4} \sim\left(d z_{4}+\cdots\right)
\end{aligned}
$$

where the ellipses represent the regular terms constructed from the seven-dimensional $\mathcal{A}_{1}^{(i)}$ and $\mathcal{A}_{0}^{(i j)}$ potentials, and we have again omitted the exponential dilatonic prefactors. In this particular example, the seven-dimensional theory does not admit a 2-charge domain wall solution, since the dot products of $\vec{b}_{134}$ and $\vec{b}_{234}$ do not satisfy (2.9).

In the previous examples, multiple axions were subjected to Scherk-Schwarz reductions, but all in the same step of dimensional reduction. Other kinds of generalisation are possible in which the Scherk-Schwarz ansatz is used at two or more different stages of a multi-step dimensional reduction procedure. For example, we may set $\mathcal{A}_{0}^{(12)}=m_{1} z_{3}$ and $\mathcal{A}_{0}^{(14)}=m_{2} z_{5}$ to obtain a massive theory in $D=6$. Configurations in $D=6$ are described in terms of $T^{1}$ bundles over $T^{4}$ in the internal space. In fact this example admits a 2-charge domain wall solution, since $\vec{b}_{123}$ and $\vec{b}_{145}$ do satsify the condition (2.9). As a final example, we may construct a massive theory in $D=4$ by setting

$$
\mathcal{A}_{0}^{(12)}=m_{1} z_{5}, \quad \mathcal{A}_{0}^{(16)}=m_{2} z_{7}, \quad \mathcal{A}_{0}^{(35)}=m_{3} z_{6}, \quad \mathcal{A}_{0}^{(45)}=m_{4} z_{7},
$$

whose internal space when lifted back to eleven dimensions has the structure of a $T^{3}$ bundle over $T^{4}$. This example allows a 4-charge domain wall solution.

\subsection{Properties of the internal space}

In this section we should like to clarify some interesting properties of the internal spaces arising in different compactification schemes. It turns out that there are a number of extra conditions to be imposed on the structure of the internal space in order to have a consistent theory. These additional requirements involve the periodicities of the internal coordinates, and the topological structure of the compactifying space.

As our first example let us consider the oxidation to $D=11$ of the domain wall solution 
obtained in section 2.1:

$$
d s_{11}^{2}=d x^{\mu} d x_{\mu}+H d y^{2}+H\left(d z_{2}^{2}+d z_{3}^{2}\right)+H^{-1}\left(d z_{1}+m z_{3} d z_{2}\right)^{2} .
$$

As we pointed out, it has the structure of a $U(1)$ principal fibre bundle, where $z_{1}$ is the fibre coordinate, while $z_{2}$ and $z_{3}$ are the coordinates on the base manifold, which in this particular case is a 2-torus. One can define a connection form

$$
\mathcal{A}_{1}^{(1)}=d z_{1}+m z_{3} d z_{2}
$$

The important property of this form is that it is globally defined. Thus not only is it invariant under coordinate transformations, but also it must give consistent periodicities for the internal coordinates. The internal space is left invariant by the following constant coordinate shifts: $z_{i} \rightarrow z_{i}+L_{i}$, where $L_{i}$ are the periods of corresponding compactifying coordinates $z_{i}$. For instance, if one shifts $z_{3}$ in formula (2.15) by $L_{3}$, then in order to keep the whole expression invariant this transformation has to be accompanied by a shift of $z_{1}$ that is given by

$$
z_{1} \rightarrow z_{1}+m L_{3} z_{2}
$$

It is clear that above expression makes sense only if the right and left-hand sides have the same transformation properties under the shift symmetry. In turn, this means that $L_{1}$ is non-trivially related to $L_{2}$ and $L_{3}$ :

$$
L_{1}=m L_{2} L_{3}
$$

Similar relations can be established for other compactification schemes. In fact in general, the period $L$ for a coordinate $z$ whose compactification yields the Kaluza-Klein 2-form field strength $\mathcal{F}_{2}$ will be

$$
L=\int \mathcal{F}_{2}
$$

Let us consider some of the other exampls that were introduced in section 2 . We already discussed the massive theory involving the simultaneous Scherk-Schwarz reduction of the three axions $\mathcal{A}_{0}^{(12)}, \mathcal{A}_{0}^{(13)}$ and $\mathcal{A}_{0}^{(123)}$. It was shown that the theory admits a 2-charge domain wall solution, which in turn can be reinterpreted from the $D=11$ point of view as a $U(1)$ principal bundle over $T^{3}$. It is manifest from the structure of the vielbeins in the compact directions that

$$
\begin{aligned}
e^{1} & \sim\left(d z_{1}+m_{1} z_{4} d z_{2}+m_{2} z_{4} d z_{3}+\ldots\right) \\
e^{i} & \sim\left(d z_{i}+\ldots\right), \quad i>1
\end{aligned}
$$


where we have omitted regular terms having no relevance to our present discussion. Analogous periodicity and consistency arguments can be given in this case also, leading to the following relations

$$
L_{1}=m_{1} L_{2} L_{4}=m_{2} L_{3} L_{4} .
$$

For a final example, we shall consider an internal space with a more complicated structure. This comes from the seven-dimensional theory obtained by performing a ScherkSchwarz reduction of $\mathcal{A}_{0}^{(13)}$ and $\mathcal{A}_{0}^{(23)}$ in $D=8$. From the eleven-dimensional point of view, all configurations in $D=7$ have the same structure for their internal spaces, namely $T^{2}$ bundles over $T^{2}$, as can be clearly seen from the form of vielbeins

$$
\begin{aligned}
& e^{1} \sim\left(d z_{1}+m_{1} z_{4} d z_{3}+\ldots\right), \quad e^{2} \sim\left(d z_{2}+m_{2} z_{4} d z_{3}+\ldots\right), \\
& e^{3} \sim\left(d z_{3}+\ldots\right), \quad e^{4} \sim\left(d z_{4}+\ldots\right)
\end{aligned}
$$

where as always we present only the relevant terms and coefficients. All the previous considerations go through without change here too. Thus similar arguments imply the following relation between the periods of the compactification coordinates

$$
L_{1}=m_{1} L_{3} L_{4}, \quad L_{2}=m_{2} L_{3} L_{4}
$$

To avoid confusion, it is should be emphasised that the compactification periods we are discussing here are not the same as the physical sizes of the compact internal dimensions. These are related to the periods by metric factors involving the dilatonic scalars, and so the actual size of the internal space is determined by the solution, rather then fixed at will or by some compactification scheme. But relations of the form (2.22) do have physical consequences, in that they impose quantisation conditions on the mass parameters. To clarify this point, let us go back to the expresssion (2.17). This formula fixes value of mass parameter in terms of periods of the compactifying coordinates,

$$
m=\frac{L_{1}}{L_{2} L_{3}} .
$$

The above relation essentially means that the mass parameter is determined, once the compactification periods are chosen. However, a more careful analysis shows that this is not precisely correct. Indeed, the assumption that the mass is simply proportional to $m_{0}$, where $m_{0}=L_{1} /\left(L_{2} L_{3}\right)$, with some integral coefficient, is good enough. It does not break any invariance, and it is consistent with the restrictions we have imposed. Obviously this argument is valid in all examples we have given in the paper. For instance from (2.22) it follows that

$$
m_{1}=n_{1} \frac{L_{1}}{L_{2} L_{4}}, \quad m_{2}=n_{2} \frac{L_{1}}{L_{3} L_{4}}
$$


where $n_{1}$ and $n_{2}$ are integers.

Thus we saw that the the domain wall charges are discretised. This discretisation of the charges are purely due to the classical effect; it is quite different from the discretisation of usual $p$-brane charges, where the existence of electric and magnetic pair will suffer a Dirac quantisation condition. Domain walls do not have electric duals (they would be (-2)-branes).

\section{$3 \quad$ Fibre bundles and generalised reductions}

In the previous section, we discussed classes of generalised reductions in which axions with global abelian shift symmetries were given a linear dependence on the compactification coordinate. In fact, as we shall explain later, one can make use of any global symmetry to allow the dependence of certain fields on the coordinates of the compactifying space. The simplest example that goes beyond the cases that we discussed previously is when the global symmetry is associated with a shift symmetry of a dilatonic scalar field. We shall present an example of such a generalised reduction in the next section. Before we go into detailed discussion of this example it is worthwhile to describe some general ideas behind all generalised reductions based on global symmetries.

Let us suppose that we have a theory in $D+1$-dimensional space-time with a Lagrangian that is left invariant by some global group $G$ of transformations. For simplicity, let us consider as an example a theory comprising a set of scalar fields $\phi^{i}$ in some representation of the group $G$, where the the Lagrangian is left invariant by the transformation

$$
\hat{\phi} \rightarrow g \hat{\phi}
$$

Here $g$ is an element of the global symmetry group $G$, and $\hat{\phi}$ is a vector with components $\phi^{i}$. One can translate this picture into the language of fibre bundles as follows. The group $G$ becomes the structure group acting on sections (i.e. the $\phi^{i}$ fields) of a trivial fibre bundle with $(D+1)$-dimensional space-time as a base manifold. The bundle is trivial because Minkowski space-time can be covered by one coordinate chart. The possibility of having a non-trivial bundle arises when one compactifies one of the spatial coordinates on a circle. Now, the space-time $M_{D+1}$ decomposes into the product $S^{1} \times M_{D}$, which certainly can be used to give a non-trivial twist in the fibres. Thus we may consider the situation where the fields $\phi^{i}$ are not globally defined over $S^{1}$, but rather are related by some element of the group $G$ when one goes from one coordinate chart to another. 
The way to implement this is to introduce the $z$-dependent factor $h(z)$ for the fields $\hat{\phi}$ in $D+1$ dimensions, so that instead of making the trivial Kaluza-Klein ansatz $\hat{\phi}(x, z)=\hat{\phi}(x)$, where $z$ are the compactifying coordinates, we impose $\hat{\phi}(x, z)=h(z) \hat{\phi}(x)$, where $h(z)$ satisfies $h\left(z_{(2)}\right)=g h\left(z_{(1)}\right)$. Here $g$ is some group element in $G$, and $z_{(1)}$ and $z_{(2)}$ are respectively the coordinates on the first and second coordinate charts on $S^{1}$. In such a case one has two locally-defined sections $\hat{\phi}_{(1)}$ and $\hat{\phi}_{(2)}$, related to one another on the intersection of the two charts by the transformation $\hat{\phi}_{(2)}=g \hat{\phi}_{(1)}$. As we shall see, typically $g$ depends on parameters characterising the fibre bundle, as does the resulting $D$-dimensional theory. In more general examples, the compact space need not be $S^{1}$. Later, we shall study compactifications where it is $T^{n}$, or more complex spaces. We shall now present some examples to make this abstract discussion a little more concrete.

\section{Generalised reductions for Cremmer-Julia symmetries}

\subsection{Scherk-Schwarz reduction of the dilaton}

Unlike the shift symmetries of axions, these transformations must be accompanied by appropriate rescalings of the higher-degree tensor fields in the theory. Consider, for example, the type IIA theory in ten dimensions, for which the bosonic Lagrangian takes the form

$\mathcal{L}=e R-\frac{1}{2} e\left(\partial \phi_{1}\right)^{2}-\frac{1}{48} e e^{-\frac{1}{2} \phi_{1}} F_{4}^{2}-\frac{1}{12} e e^{\phi_{1}}\left(F_{3}^{(1)}\right)^{2}-\frac{1}{4} e e^{-\frac{3}{2} \phi_{1}}\left(\mathcal{F}_{2}^{(1)}\right)^{2}+\frac{1}{2} *\left(d A_{3} \wedge d A_{3} \wedge A_{2}^{(1)}\right)$,

where $F_{4}=d A_{3}-d A_{2}^{(1)} \wedge \mathcal{A}_{1}^{(1)}, F_{3}^{(1)}=d A_{2}^{(1)}$ and $\mathcal{F}_{2}^{(1)}=d \mathcal{A}_{1}^{(1)}$. This is invariant under the following global shift transformation of the dilaton:

$$
\phi_{1} \longrightarrow \phi_{1}+c, \quad \mathcal{A}_{1}^{(1)} \longrightarrow e^{\frac{3}{4} c} \mathcal{A}_{1}^{(1)}, \quad A_{2}^{(1)} \longrightarrow e^{-\frac{1}{2} c} A_{2}^{(1)}, \quad A_{3} \longrightarrow e^{\frac{1}{4} c} A_{3}
$$

Clearly one can transform to variables in which the dilaton is covered by a derivative everywhere, by redefining each gauge potential $A$, with kinetic term $e^{a \phi} F^{2}$, according to $A \rightarrow e^{-\frac{1}{2} a \phi} A$. Equivalently, we may simply make an appropriate generalised reduction ansatz on the original variables, of the form

$$
\begin{aligned}
\phi_{1}(x, z) & \longrightarrow \phi_{1}(x)+m z \\
\mathcal{A}_{1}^{(1)}(x, z) & \longrightarrow e^{\frac{3}{4} m z}\left(\mathcal{A}_{1}^{(1)}(x)+\mathcal{A}_{0}^{(12)}(x) \wedge d z\right) \\
A_{2}^{(1)}(x, z) & \longrightarrow e^{-\frac{1}{2} m z}\left(A_{2}^{(1)}(x)+A_{1}^{(12)}(x) \wedge d z\right), \\
A_{3}(x, z) & \longrightarrow e^{\frac{1}{4} m z}\left(A_{3}(x)+A_{2}^{(2)}(x) \wedge d z\right)
\end{aligned}
$$


This corresponds to setting the global parameter $c$ in (4.2) equal to $m z$, and then making the usual $z$-independent reduction ansatz on the transformed fields. Because of the shift symmetry, it is guaranteed that if we substitute (4.3), together with the standard KaluzaKlein ansatz for the metric, into the ten-dimensional equations of motion following from (4.1), the resulting equations will be independent of the compactification coordinate $z$, and will correspond to a consistent truncation to $D=9$. Accordingly, we may instead consistently substitute the ansatz into the ten-dimensional Lagrangian, to obtain the ninedimensional result. This will be very similar to the form of the usual massless supergravity, given by (A.1) with $D=9$, except that now some of the field strengths have acquired extra terms involving the mass parameter $m$, and also there will be a cosmological term, given by

$$
\mathcal{L}_{\text {cosmo }}=-\frac{1}{2} m^{2} e^{\frac{4}{\sqrt{7}} \phi_{2}} .
$$

The field strengths have the form

$$
\begin{aligned}
& \mathcal{F}_{1}^{(12)}=d \mathcal{A}_{0}^{(12)}-\frac{3}{4} m \mathcal{A}_{1}^{(1)}, \quad \mathcal{F}_{2}^{(1)}=d \mathcal{A}_{1}^{(1)}+\cdots, \\
& F_{2}^{(12)}=d A_{1}^{(12)}+\frac{1}{2} m A_{2}^{(1)}, \quad F_{3}^{(1)}=d A_{2}^{(1)}+\cdots, \\
& F_{3}^{(2)}=d A_{2}^{(2)}-\frac{1}{4} m A_{3}+\cdots, F_{4}=d A_{3}+\cdots, \\
& \mathcal{F}_{2}^{(2)}=d \mathcal{A}_{1}^{(2)},
\end{aligned}
$$

where the ellipses represent higher-order terms associated with the Kaluza-Klein modifications. It is clear from these expressions that the fields $\mathcal{A}_{1}^{(1)}, A_{2}^{(1)}$ and $A_{3}$ become massive,

eating in the process the fields $\mathcal{A}_{0}^{(12)}, A_{1}^{(12)}$ and $A_{2}^{(2)}$ respectively. It is interesting to note also that the coupling of the dilatonic scalar $\phi_{2}$ in the cosmological term (4.4) is characterised in terms of the parameter $\Delta$, defined in [19], by $\Delta=0$.

\section{2 $S L(2, \mathbb{R})$ Scherk-Schwarz reduction in type IIB}

In this subsection, we shall discuss a Scherk-Schwarz reduction that makes use of the global $S L(2, R)$ symmetry of the type IIB supergravity theory. This illustrates a procedure that can be applied in a more general context, for any global symmetry.

Following the notation in [20], we may write a Lagrangian for a bosonic subsector of the type IIB theory, where the self-dual 5 -form is set to zero. Since this is a singlet under $S L(2, R)$, it is not in any case of relevance in our discussion. The Lagrangian takes the form

$$
\mathcal{L}=e R+\frac{1}{4} e \operatorname{tr}\left(\partial \mathcal{M} \partial \mathcal{M}^{-1}\right)-\frac{1}{12} e H_{3}^{T} \mathcal{M} H_{3}
$$


where $H_{3}=\left(\begin{array}{c}H_{3}^{(1)} \\ H_{3}^{(2)}\end{array}\right)$, with $H_{3}^{(1)}$ and $H_{3}^{(2)}$ being the NS-NS and R-R 3-forms. $\mathcal{M}$ is the matrix

$$
\mathcal{M}=e^{\phi}\left(\begin{array}{cc}
|\tau|^{2} & \chi \\
\chi & 1
\end{array}\right)
$$

where $\tau=\chi+i e^{-\phi}$, and $\chi$ and $\phi$ are the axion and dilaton. The Lagrangian is invariant under the global $S L(2, R)$ transformation

$$
\mathcal{M} \longrightarrow \Lambda \mathcal{M} \Lambda^{T}, \quad B_{2} \longrightarrow\left(\Lambda^{T}\right)^{-1} B_{2}
$$

where $H_{3}^{(i)}=d B_{2}^{(i)}$. Let us introduce a matrix $\Lambda$, which is effectively the "square root" of $\mathcal{M}$, such that $\mathcal{M}=\Lambda^{T} \Lambda$. One can, for example, take

$$
\Lambda=\left(\begin{array}{cc}
e^{-\frac{1}{2} \phi} & 0 \\
\chi e^{\frac{1}{2} \phi} & e^{\frac{1}{2} \phi}
\end{array}\right)
$$

Let us now perform a generalised dimensional reduction on a circle, where $z$ dependence is introduced in the following way:

$$
\begin{aligned}
\mathcal{M}(x, z) & =\lambda(z)^{T} \mathcal{M}(x) \lambda(z) \\
B_{2}(x, z) & =\lambda(z)^{-1}\left(B_{2}(x)+B_{1}(x) \wedge d z\right),
\end{aligned}
$$

together with the standard Kaluza-Klein ansatz for the metric. The $S L(2, R)$ matrix $\lambda(z)$ is required to have the following property:

$$
\left(\partial_{z} \lambda(z)\right) \lambda(z)^{-1}=C
$$

where $C$ is a constant matrix of the form

$$
C=\left(\begin{array}{cc}
\frac{1}{2} m_{1} & 0 \\
m_{2} & -\frac{1}{2} m_{1}
\end{array}\right)
$$

with $m_{1}$ and $m_{2}$ being arbitrary constants. Substituting into (4.6), we see that the tendimensional Lagrangian reduces to

$$
\begin{aligned}
\mathcal{L}_{9}= & e R-\frac{1}{2} e(\partial \varphi)^{2}+\frac{1}{4} e \operatorname{tr}\left(\partial \mathcal{M} \partial \mathcal{M}^{-1}\right)-\frac{1}{4} e e^{-\frac{4}{\sqrt{7}} \varphi} \mathcal{F}_{2}^{2}-\frac{1}{12} e e^{-\frac{1}{\sqrt{7}} \varphi} H_{3}^{T} \mathcal{M} H_{3} \\
& -\frac{1}{4} e e^{\frac{3}{\sqrt{7}} \varphi} H_{2}^{T} \mathcal{M} H_{2}-\frac{1}{2} e e^{\frac{4}{\sqrt{7}} \varphi} \operatorname{tr}\left(C^{2}+C^{T} \mathcal{M} C \mathcal{M}^{-1}\right)
\end{aligned}
$$

where $\varphi$ and $\mathcal{F}_{2}=d \mathcal{A}_{1}$ come from the dimensional reduction of the metric. The ninedimensional 3 -form and 2-form field strengths are given by

$$
H_{3}=d B_{2}-H_{2} \wedge \mathcal{A}_{1}, \quad H_{2}=d B_{1}-C B_{2},
$$


showing that under appropriate choices for $C$, the fields $B_{2}$ become massive, eating $B_{1}$ in the process. Note that the "cosmological terms" described by the final expression in (4.13) take the form

$$
\mathcal{L}_{\text {cosmo }}=-\frac{1}{2} m_{1}^{2} e e^{\frac{4}{\sqrt{7}} \varphi}-\frac{1}{2} e\left(m_{2}+m_{1} \chi\right)^{2} e^{2 \phi+\frac{4}{\sqrt{7}} \varphi}
$$

in terms of $\phi$ and $\chi$. In fact when $m_{1} \neq 0$, the latter term is actually a mass term for $\chi$.

The $z$ dependence of the ten-dimensional scalar fields $\phi(x, z)$ and $\chi(x, z)$ is dictated by the structure of the $z$-dependent matrix $\lambda(z)$, together with the ansatz for $\mathcal{M}(x, z)$ given in (4.10). This can be re-expressed in the form $\Lambda(x, z)=\Lambda(x) \lambda(z)$, where $\mathcal{M}(x)=\Lambda(x)^{T} \Lambda(x)$. The matrix $\lambda(z)$ can be written as

$$
\lambda(z)=e^{z C}=\left(\begin{array}{cc}
e^{\frac{1}{2} m_{1} z} & 0 \\
\frac{2 m_{2}}{m_{1}} \sinh \left(\frac{1}{2} m_{1} z\right) & e^{-\frac{1}{2} m_{1} z}
\end{array}\right) .
$$

From this, it follows that the ten-dimensional fields must have $z$ dependence given by

$$
\begin{aligned}
& \phi(x, z)=\phi(x)-m_{1} z \\
& \chi(x, z)=e^{m_{1} z} \chi(x)+\frac{m_{2}}{m_{1}}\left(e^{m_{1} z}-1\right) .
\end{aligned}
$$

\section{3 $S L(2, R)$ Scherk-Schwarz reduction in type IIA}

If the type IIA theory is reduced to nine dimensions, it also has an $S L(2, R)$ global symmetry. This can be used for performing a Scherk-Schwarz reduction, in the same way as we did for the type IIB theory above. The advantage of looking at this example is that the $S L(2, R)$ can be given a clear geometric origin, by lifting the theory up to eleven dimensions. (The analogous F-theory origin of the $S L(2, R)$ symmetry in the type IIB theory is not so well understood.) In the discussion that follows, we shall focus on the subsector of the theory that is relevant for describing domain-wall solutions in the Scherk-Schwarz reduced eightdimensional theory. This subsector is obtained from the Scherk-Schwarz reduction of $\mathcal{L}=$ $e R+\frac{1}{4} e \operatorname{tr}\left(\partial \mathcal{M} \partial \mathcal{M}^{-1}\right)$, exactly as in the type IIB example above. Thus the relevant part of the eight-dimensional Lagrangian will be

$$
\begin{aligned}
\mathcal{L}_{8}= & e R-\frac{1}{2} e(\partial \varphi)^{2}+\frac{1}{4} e \operatorname{tr}\left(\partial \mathcal{M} \partial \mathcal{M}^{-1}\right) \\
& -\frac{1}{2} m_{1}^{2} e e^{\sqrt{\frac{7}{3}} \varphi}-\frac{1}{2} e\left(m_{2}+m_{1} \chi\right)^{2} e^{2 \phi+\sqrt{\frac{7}{3} \varphi}} .
\end{aligned}
$$

This admits two domain wall solutions, corresponding to either $m_{1}=0, m_{2} \neq 0$, or $m_{2}=0$, $m_{1} \neq 0$. The former has a dilaton coupling described by $\Delta=4$, and has a solution of the 
standard form [21, []

$$
\begin{aligned}
d s_{8}^{2} & =H^{1 / 6} d x^{\mu} d x_{\mu}+H^{7 / 6} d y^{2} \\
e^{\phi} & =H^{\sqrt{3 / 19}}, \quad e^{\varphi}=H^{\frac{1}{2} \sqrt{7 / 19}} .
\end{aligned}
$$

where $H=1+m_{2}|y|$. The latter gives a solution with $\Delta=0$. After oxidation back to $D=11$, the solution (4.19) gives rise to the metric

$$
d s_{11}^{2}=d s_{8}^{2}+H d y^{2}+H d z_{3}^{2}+H e^{m_{1} z_{3}} d z_{2}^{2}+H^{-1} e^{-m_{1} z_{3}} d z_{1}^{2} .
$$

The three-dimensional manifold described by coordinates $\left(z_{1}, z_{2}, z_{3}\right)$ has the structure of an homogeneous space, as can be easily shown from the fact that at a fixed value of the coordinate $y$ the vielbeins $e^{1}=\lambda^{-1} e^{-\frac{m_{1}}{2} z_{3}} d z_{1}, e^{2}=\lambda e^{\frac{m_{1}}{2} z_{3}} d z_{2}$, and $e^{3}=\lambda d z_{3}$ satisfy

$$
d e^{1}=-\frac{m_{1}}{2 \lambda} e^{3} \wedge e^{1}, \quad d e^{2}=\frac{m_{1}}{2 \lambda} e^{3} \wedge e^{2}, \quad d e^{3}=0 .
$$

The spin connection and curvature 2-form are therefore given by

$$
\begin{aligned}
& \omega_{12}=0, \quad \omega_{23}=\frac{m_{1}}{2 \lambda} e^{2}, \quad \omega_{31}=\frac{m_{1}}{2 \lambda} e^{2}, \\
& \Theta_{12}=\frac{m_{1}^{2}}{4 \lambda^{2}} e^{1} \wedge e^{2}, \quad \Theta_{23}=-\frac{m_{1}^{2}}{4 \lambda^{2}} e^{2} \wedge e^{3}, \quad \Theta_{31}=-\frac{m_{1}^{2}}{4 \lambda^{2}} e^{3} \wedge e^{1} .
\end{aligned}
$$

In this case, the metric is of type $\mathrm{V}$ in the Bianchi classification scheme. It is worth mentioning that all solutions of eight-dimensional supergravity theory will preserve the structure of this metric on the internal space.

\section{Generalised reduction from $D=11$}

As another example of a more general kind of Scherk-Schwarz reduction, we may take eleven-dimensional supergravity as a starting point. The bosonic Lagrangian takes the form

$$
\mathcal{L}=\hat{e} \hat{R}-\frac{1}{48} e \hat{F}_{4}^{2}-\frac{1}{6} *\left(\hat{F}_{4} \wedge \hat{F}_{4} \wedge \hat{A}_{3}\right)
$$

where $\hat{F}_{4}=d \hat{A}_{3}$. The theory has an homogeneous global scaling symmetry, under which the fields $\hat{g}_{M N}$ and $\hat{A}_{M N P}$ undergo the constant rescalings

$$
\hat{g}_{M N} \longrightarrow \lambda^{2} \hat{g}_{M N}, \quad \hat{A}_{M N P} \longrightarrow \lambda^{3} \hat{A}_{M N P} .
$$

Although the action is not invariant under this transformation, the equations of motion, namely

$$
\begin{aligned}
\hat{R}_{M N} & =\frac{1}{12}\left(\hat{F}_{M P Q R} \hat{F}_{N}^{P Q R}-\frac{1}{12} \hat{F}_{4}^{2} \hat{g}_{M N}\right), \\
\hat{\nabla}_{M} \hat{F}^{M N P Q} & =\frac{1}{1152} \epsilon^{N P Q R_{1} \cdots R_{8}} \hat{F}_{R_{1} \cdots R_{4}} \hat{F}_{R_{5} \cdots R_{8}}
\end{aligned}
$$


are invariant, by virtue of the fact that $(5.2)$ gives an homogeneous scaling of the entire action. Note that the equation of motion for $\hat{F}_{4}$ may be written more elegantly as

$$
d * \hat{F}_{4}=\frac{1}{2} \hat{F}_{4} \wedge \hat{F}_{4}
$$

Let us now perform a dimensional reduction to $D=10$ in which the eleven-dimensional fields are allowed dependences on the compactifying coordinate $z$ of the form

$$
\begin{aligned}
d \hat{s}^{2} & =e^{2 m z+\frac{1}{6} \varphi} d s^{2}+e^{2 m z-\frac{4}{3} \varphi}\left(d z+\mathcal{A}_{1}\right)^{2} \\
\hat{A}_{3} & =e^{3 m z} A_{3}+e^{3 m z} A_{2} \wedge d z
\end{aligned}
$$

where the ten-dimensional fields on the right-hand sides are independent of $z$. It is evident that if these expressions are substituted into the eleven-dimensional equations of motion the $z$-dependence will cancel, owing to the global scaling invariance (5.2), and thus we will obtain a set of ten-dimensional equations of motion that are a consistent truncation of the eleven-dimensional equations.

The computations are facilitated by first performing a Weyl rescaling in $D=11$, and relating the Ricci curvature $\hat{R}_{M N}$ of the metric $\hat{g}_{M N}$ to that of a Weyl rescaled metric $\tilde{g}_{M N}$ for which $\hat{g}_{M N}=e^{2 \sigma} \tilde{g}_{M N}$. In $n$ dimensions, a simple calculation gives

$$
\hat{R}_{M N}=\widetilde{R}_{M N}+(n-2)\left(\partial_{M} \sigma \partial_{N} \sigma-\widetilde{\nabla}_{M} \partial_{N} \sigma-\tilde{g}^{P Q} \partial_{P} \sigma \partial_{Q} \sigma \tilde{g}_{M N}\right)-\square \sigma \tilde{g}_{M N}
$$

With the help of this equation, it is straightforward to substitute the reduction ansätze (5.5) into the eleven-dimensional equations of motion (5.3), to obtain the following tendimensional equations of motion:

$$
\begin{aligned}
& \square \varphi=-\frac{3}{8} e^{-\frac{3}{2} \varphi} \mathcal{F}_{2}^{2}+\frac{1}{12} e^{\varphi} F_{3}^{2}-\frac{1}{96} e^{-\frac{1}{2} \varphi} F_{4}^{2}+\frac{27}{2} m^{2} \mathcal{A}_{\mu} \mathcal{A}^{\mu}+9 m \mathcal{A}^{\mu} \partial_{\mu} \varphi-\frac{3}{2} m \nabla_{\mu} \mathcal{A}^{\mu}, \\
& R_{\mu \nu}-\frac{1}{2} R g_{\mu \nu}=\frac{1}{2}\left(\partial_{\mu} \varphi \partial_{\nu} \varphi-\frac{1}{2}(\partial \varphi)^{2} g_{\mu \nu}\right)+\frac{1}{2} e^{-\frac{3}{2} \varphi}\left(\mathcal{F}_{\mu \rho} \mathcal{F}_{\nu} \rho-\frac{1}{4} \mathcal{F}_{2}^{2} g_{\mu \nu}\right) \\
& +\frac{1}{4} e^{\varphi}\left(F_{\mu \rho \sigma} F_{\nu}^{\rho \sigma}-\frac{1}{6} F_{3}^{2} g_{\mu \nu}\right)+\frac{1}{12} e^{-\frac{1}{2} \varphi}\left(F_{\mu \rho \sigma \lambda} F_{\nu}{ }^{\rho \sigma \lambda}-\frac{1}{8} F_{4}^{2} g_{\mu \nu}\right) \\
& -9 m^{2}\left(\mathcal{A}_{\mu} \mathcal{A}_{\nu}+4 \mathcal{A}_{\rho} \mathcal{A}^{\rho} g_{\mu \nu}\right)-36 m^{2} e^{\frac{3}{2} \varphi} g_{\mu \nu} \\
& -\frac{9}{2} m\left(\nabla_{\mu} \mathcal{A}_{\nu}+\nabla_{\nu} \mathcal{A}_{\mu}-2 \nabla_{\rho} \mathcal{A}^{\rho} g_{\mu \nu}\right) \\
& +\frac{3}{4} m\left(\mathcal{A}_{\mu} \partial_{\nu} \varphi+\mathcal{A}_{\nu} \partial_{\mu} \varphi-\mathcal{A}^{\rho} \partial_{\rho} \varphi g_{\mu \nu}\right), \\
& \nabla_{\nu}\left(e^{-\frac{3}{2} \varphi} \mathcal{F}_{\mu}{ }^{\nu}\right)=12 m \partial_{\mu} \varphi+18 m^{2} \mathcal{A}_{\mu}+9 m e^{-\frac{3}{2} \varphi} \mathcal{A}^{\nu} \mathcal{F}_{\mu \nu}-\frac{1}{6} e^{-\frac{1}{2} \varphi} F_{\mu \nu \rho \sigma} F^{\nu \rho \sigma}, \\
& \nabla^{\sigma}\left(e^{-\frac{1}{2} \varphi} F_{\mu \nu \rho \sigma}\right)=-6 m e^{\varphi} F_{\mu \nu \rho}+6 m e^{-\frac{1}{2} \varphi} \mathcal{A}^{\sigma} F_{\mu \nu \rho \sigma}-\frac{1}{144} \epsilon_{\mu \nu \rho \sigma_{1} \ldots \sigma_{7}} F^{\sigma_{1} \sigma_{2} \sigma_{3} \sigma_{4}} F^{\sigma_{5} \sigma_{6} \sigma_{7}} \text {, } \\
& \nabla^{\sigma}\left(e^{\varphi} F_{\mu \nu \sigma}\right)=6 m e^{\varphi} \mathcal{A}^{\sigma} F_{\mu \nu \sigma}+\frac{1}{2} e^{-\frac{1}{2} \varphi} F_{\mu \nu \sigma \rho} \mathcal{F}^{\sigma \rho}+\frac{1}{1152} \epsilon_{\mu \nu \rho_{1} \ldots \rho_{8}} F^{\rho_{1} \rho_{2} \rho_{3} \rho_{4}} F^{\rho_{5} \rho_{6} \rho_{7} \rho_{8}} .
\end{aligned}
$$


Note that the last three equations can be concisely written using the language of differential forms as follows

$$
\begin{aligned}
& d\left(e^{-\frac{3}{2} \varphi} * \mathcal{F}_{2}\right)=-12 m * d \varphi-18 m^{2} * \mathcal{A}_{1}+9 m e^{-\frac{3}{2} \varphi} \mathcal{A}_{1} \wedge * \mathcal{F}_{2}-e^{-\frac{1}{2} \varphi} F_{3} \wedge * F_{4} \\
& d\left(e^{-\frac{1}{2} \varphi} * F_{4}\right)=6 m e^{\varphi} * F_{3}+6 m e^{-\frac{1}{2} \varphi} \mathcal{A}_{1} \wedge * F_{4}+F_{4} \wedge F_{3} \\
& d\left(e^{\varphi} * F_{3}\right)=6 m e^{\varphi} \mathcal{A}_{1} \wedge * F_{3}-e^{-\frac{1}{2} \varphi} \mathcal{F}_{2} \wedge * F_{4}+\frac{1}{2} F_{4} \wedge F_{4} .
\end{aligned}
$$

Here, the field strengths in $D=10$ are defined by

$$
\begin{aligned}
\mathcal{F}_{2} & =d \mathcal{A}_{1} \\
F_{3} & =d A_{2}-3 m A_{3} \\
F_{4} & =d A_{3}-d A_{2} \wedge \mathcal{A}_{1}+3 m A_{3} \wedge \mathcal{A}_{1} .
\end{aligned}
$$

Note that the calculation of the field equations for $F_{3}$ and $F_{4}$ can be simplified by first establishing the following lemmata:

$$
\begin{aligned}
\hat{*} X_{n} & =(-1)^{n} e^{(11-2 n) m z-\frac{1}{6}(n-1) \varphi_{*} X_{n} \wedge\left(d z+\mathcal{A}_{1}\right),} \\
\hat{*}\left(X_{n} \wedge\left(d z+\mathcal{A}_{1}\right)\right) & =e^{(9-2 n) m z+\frac{1}{6}(9-n) \varphi} * X_{n}
\end{aligned}
$$

where $X_{n}$ is any $n$-form living in the ten-dimensional base manifold, and $\hat{*}$ and $*$ denote the Hodge duals in $D=11$ and $D=10$ respectively. Our conventions for the definition of the Hodge dual in $D$ dimensions are as follows:

$$
\begin{aligned}
*\left(d x^{\mu_{1}} \cdots d x^{\mu_{p}}\right) & =\frac{1}{q !} \epsilon_{\nu_{1} \cdots \nu_{q}}{ }^{\mu_{1} \cdots \mu_{p}} d x^{\nu_{1}} \cdots d x^{\nu_{q}}, \\
(* \omega)_{\mu_{1} \cdots \mu_{q}} & =\frac{1}{p !} \epsilon_{\mu_{1} \cdots \mu_{q}}{ }^{\nu_{1} \cdots \nu_{p}} \omega_{\nu_{1} \cdots \nu_{p}}
\end{aligned}
$$

where $q=D-p$, and $\omega$ is a $p$-form. These imply that

$$
\begin{aligned}
* * \omega & =(-1)^{p q-1} \omega, \\
(* \omega) \wedge \omega & =-\frac{1}{p !}|\omega|^{2} d^{D} x,
\end{aligned}
$$

where $\omega$ is any $p$-form, $|\omega|^{2}=\omega_{\mu_{1} \cdots \mu_{p}} \omega^{\mu_{1} \cdots \mu_{p}}$, and $d^{D} x=(D !)^{-1} \epsilon_{\mu_{1} \cdots \mu_{D}} d x^{\mu_{1}} \cdots d x^{D}$ is the volume form. (Had the signature been Euclidean, rather than Lorentzian, the right-hand sides of the two equations in (5.12) would each be multiplied by a further $(-1)$ factor.)

The massive ten-dimensional theory that we have obtained here is essentially the same as the one obtained in [13] (except that in [13] the 4-form field strength was set to zero before making the reduction.) The procedure by which we have obtained the ten-dimensional theory is slightly different from the one used in [13]; in that paper, the eleven-dimensional 
supergravity starting point was modified slightly, by solving the superspace constraints with a conformal spin connection [22] rather than the usual one. The modified eleven-dimensional theory was then reduced to ten dimensions using ordinary Kaluza-Klein reduction. In our case, by contrast, the eleven-dimensional starting point was the usual one, and the dimensional reduction was modified to the Scherk-Schwarz scheme. As was observed in [13], the equations of motion (5.8) for this massive theory cannot be derived from a Lagrangian. This is understandable, in view of the fact that we derived the ten-dimensional equations by means of a truncation of the eleven-dimensional theory which, although consistent, could be performed only at the level of the equations of motion, since it involved the use of a global transformation that is a symmetry of the equations of motion, but not of the action.

It can be easily seen that the equations (5.8) possess the following set of local symmetries:

$$
\begin{aligned}
& \varphi \rightarrow \varphi+\lambda, \quad \mathcal{A}_{1} \rightarrow \mathcal{A}_{1}-\frac{2}{3 m} d \lambda, \quad g_{\mu \nu} \rightarrow e^{-\frac{3}{2} \lambda} g_{\mu, \nu}, \\
& A_{2} \rightarrow e^{-2 \lambda} A_{2}, \quad A_{3} \rightarrow e^{-2 \lambda}\left(A_{3}-\frac{2}{3 m} d \lambda \wedge A_{2}\right)
\end{aligned}
$$

and also

$$
\begin{aligned}
& A_{2} \rightarrow A_{2}+d \lambda_{1}+\lambda_{2}, \\
& A_{3} \rightarrow A_{3}+\frac{1}{3 m} d \lambda_{2},
\end{aligned}
$$

where $\lambda, \lambda_{1}$ and $\lambda_{2}$ are arbitrary 0-form, 1-form and 2-form parameters respectively. Note in particular that the parameters $\lambda$ and $\lambda_{2}$ describe Stückelberg shift symmetries of $\varphi$ and $A_{2}$ respectively. This is a characteristic feature of massive theories, where the extra longitudinal degrees of freedom of the massive fields $\left(\mathcal{A}_{1}\right.$ and $\left.A_{3}\right)$ are replaced by additional fields ( $\varphi$ and $A_{2}$ ), together with Stückelberg gauge transformations that can be used to eliminate these extra fields. Indeed, one can choose a gauge such that $\varphi=0$ and $A_{2}=d \lambda_{1}$, by choosing $\lambda=-\varphi$ and $\lambda_{2}=-A_{2}$. In this gauge, the set of equations (5.8) reduces to the form

$$
\begin{gathered}
3 m d * \mathcal{A}_{1}=\frac{3}{2} \mathcal{F}_{2} \wedge * \mathcal{F}_{2}+9 m^{2} A_{3} \wedge * A_{3}+\frac{1}{2} F_{4} \wedge * F_{4}+27 m^{2} \mathcal{A}_{1} \wedge * \mathcal{A}_{1} \\
d * \mathcal{F}_{2}=-18 m^{2} * \mathcal{A}_{1}+9 m \mathcal{A}_{1} \wedge * \mathcal{F}_{2}+3 m A_{3} \wedge * F_{4} \\
R_{\mu \nu}-\frac{1}{2} R g_{\mu \nu}=\frac{1}{2}\left(\mathcal{F}_{\mu \rho} \mathcal{F}_{\nu}{ }^{\rho}-\frac{1}{4} \mathcal{F}_{2}^{2} g_{\mu \nu}\right) \\
+\frac{9}{4} m^{2}\left(A_{\mu \rho \sigma} A_{\nu}{ }^{\rho \sigma}-\frac{1}{6} A_{3}^{2} g_{\mu \nu}\right)+\frac{1}{12}\left(F_{\mu \rho \sigma \lambda} F_{\nu}{ }^{\rho \sigma \lambda}-\frac{1}{8} F_{4}^{2} g_{\mu \nu}\right) \\
-9 m^{2}\left(\mathcal{A}_{\mu} \mathcal{A}_{\nu}+4 \mathcal{A}_{\rho} \mathcal{A}^{\rho} g_{\mu \nu}\right)-36 m^{2} g_{\mu \nu} \\
-\frac{9}{2} m\left(\nabla_{\mu} \mathcal{A}_{\nu}+\nabla_{\nu} \mathcal{A}_{\mu}-2 \nabla_{\rho} \mathcal{A}^{\rho} g_{\mu \nu}\right) \\
d * F_{4}=-18 m^{2} * A_{3}+6 m \mathcal{A}_{1} \wedge * F_{4}-3 m F_{4} \wedge A_{3} \\
3 m d * A_{3}=18 m^{2} \mathcal{A}_{1} \wedge * A_{3}+\mathcal{F}_{2} \wedge * F_{4}-\frac{1}{2} F_{4} \wedge F_{4}
\end{gathered}
$$


where now $F_{4}=d A_{3}+3 m A_{3} \wedge \mathcal{A}_{1}$. The first and last equations here, which were originally the equations of motion for the fields $\varphi$ and $A_{2}$ that have been gauged to zero, play the rôle of constraints on the remaining fields, and are not independent of the other equations. Specifically, the first equation can be obtained by differentiation of the second, accompanied by appropriate resubstitutions of the other field equations. Similarly, the final equation can be derived by differentiation of the equation of motion for the 3-form potential, followed by appropriate resubstitutions from the equations of motion. Eventually, therefore, we are left with a theory of massive 1 -form and 3 -form potentials, coupled to gravity.

It is of interest to study solutions of this massive ten-dimensional theory. In [13], it was shown that, unlike the Romans theory, this massive supergravity does do admit an 8-brane domain-wall solution. It was shown, however, that it admits a time-dependent BPS solution that preserves $\frac{1}{2}$ the supersymmetry [13]. Here, we observe that there is another simple solution to the equations of motion (5.15), in which the gauge fields $A_{3}$ and $\mathcal{A}_{1}$ are set to zero. The only remaining non-trivial equation in (5.15) is the Einstein equation, which reduces to

$$
R_{\mu \nu}=9 m^{2} g_{\mu \nu}
$$

This admits a de Sitter spacetime solution. We may re-interpret this as a solution in eleven dimensions, by reversing the steps of the generalised dimensional reduction. Thus from (5.5), we find that the metric in $D=11$ takes the form

$$
\begin{aligned}
d s_{11}^{2} & =e^{2 m z} d z^{2}+e^{2 m z} d s_{10}^{2} \\
& =d \rho^{2}+m^{2} \rho^{2} d s_{10}^{2}
\end{aligned}
$$

where $\rho=e^{m z} / m$. Since the de Sitter metric $d s_{10}^{2}$ satisfies (5.16), it follows that it can be written as $d s_{10}^{2}=m^{-2} d \Omega_{10}^{2}$, where $d \Omega_{10}^{2}$ is the metric on a "unit radius" de Sitter spacetime whose Riemann tensor is given by $R_{\mu \nu \rho \sigma}=g_{\mu \rho} g_{\nu \sigma}-g_{\mu \sigma} g_{\nu \rho}$, and so we have

$$
d s_{11}^{2}=d \rho^{2}+\rho^{2} d \Omega_{10}^{2} .
$$

This is in fact a metric on flat space. This can be seen from the fact that if $d \Omega_{10}^{2}$ were the metric on the unit-radius 10-sphere, the metric (5.18) would be nothing but flat space in hyperspherical polar coordinates. The de Sitter metric is obtained from the 10-sphere metric by Lorentzianising one of the coordinates, but this will not affect the results for local curvature calculations, and so the Riemann tensor for (5.18) vanishes. 


\section{Acknowledgement}

We are grateful to E. Cremmer, B. Julia fand K.S. Stelle for discussions on non-compact global symmetries in supergravity.

\section{A Lagrangian of $D$-dimensional maximal supergravity}

In this appendix, we present the bosonic sector of the Lagrangian for $D$-dimensional maximal supergravity, obtained by dimensional reduction from $D=11$. The notation and conventions are those of 16 .

$$
\begin{aligned}
\mathcal{L}= & e R-\frac{1}{2} e(\partial \vec{\phi})^{2}-\frac{1}{48} e e^{\vec{a} \cdot \vec{\phi}} F_{4}^{2}-\frac{1}{12} e \sum_{i} e^{\vec{a}_{i} \cdot \vec{\phi}}\left(F_{3}^{i}\right)^{2}-\frac{1}{4} e \sum_{i<j} e^{\vec{a}_{i j} \cdot \vec{\phi}}\left(F_{2}^{i j}\right)^{2} \\
& -\frac{1}{4} e \sum_{i} e^{\vec{b}_{i} \cdot \vec{\phi}}\left(\mathcal{F}_{2}^{i}\right)^{2}-\frac{1}{2} e \sum_{i<j<k} e^{\vec{a}_{i j k} \cdot \vec{\phi}}\left(F_{1}^{i j k}\right)^{2}-\frac{1}{2} e \sum_{i<j} e^{\vec{b}_{i j} \cdot \vec{\phi}}\left(\mathcal{F}_{1}^{i j}\right)^{2}+\mathcal{L}_{F F A},
\end{aligned}
$$

where the "dilaton vectors" $\vec{a}, \vec{a}_{i}, \vec{a}_{i j}, \vec{a}_{i j k}, \vec{b}_{i}, \vec{b}_{i j}$ are constants that characterise the couplings of the dilatonic scalars $\vec{\phi}$ to the various gauge fields. They are given by [16]

$$
\begin{array}{lll} 
& F_{M N P Q} & \text { vielbein } \\
4-\text { form : } & \vec{a}=-\vec{g}, & \\
3-\text { forms : } & \vec{a}_{i}=\overrightarrow{f_{i}}-\vec{g}, & \\
2-\text { forms : } & \vec{a}_{i j}=\vec{f}_{i}+\overrightarrow{f_{j}}-\vec{g}, & \vec{b}_{i}=-\vec{f}_{i}, \\
1-\text { forms : } & \vec{a}_{i j k}=\overrightarrow{f_{i}}+\overrightarrow{f_{j}}+\overrightarrow{f_{k}}-\vec{g}, & \vec{b}_{i j}=-\vec{f}_{i}+\vec{f}_{j}, \\
0-\text { forms : } & \vec{a}_{i j k \ell}=\vec{f}_{i}+\vec{f}_{j}+\vec{f}_{k}+\overrightarrow{f_{\ell}}-\vec{g}, & \vec{b}_{i j k}=-\vec{f}_{i}+\vec{f}_{j}+\vec{f}_{k}
\end{array}
$$

where the vectors $\vec{g}$ and $\vec{f}_{i}$ have $(11-D)$ components in $D$ dimensions, and are given by

$$
\begin{aligned}
\vec{g} & =3\left(s_{1}, s_{2}, \ldots, s_{11-D}\right) \\
\overrightarrow{f_{i}} & =(\underbrace{0,0, \ldots, 0}_{i-1},(10-i) s_{i}, s_{i+1}, s_{i+2}, \ldots, s_{11-D})
\end{aligned}
$$

where $s_{i}=\sqrt{2 /((10-i)(9-i))}$. It is easy to see that they satisfy

$$
\vec{g} \cdot \vec{g}=\frac{2(11-D)}{D-2}, \quad \vec{g} \cdot \overrightarrow{f_{i}}=\frac{6}{D-2}, \quad \overrightarrow{f_{i}} \cdot \overrightarrow{f_{j}}=2 \delta_{i j}+\frac{2}{D-2} .
$$

We have also included the dilaton vectors $\vec{a}_{i j k \ell}$ and $\vec{b}_{i j k}$ for "0-form field strengths" in (A.2), although they do not appear in (A.1), because they fit into the same general pattern and they do arise in the generalised reduction procedures for axions that we consider in section 2 in this paper [1, 2, 3, 4, 5, 6]. 
The field strengths are given by

$$
\begin{aligned}
F_{4} & =\tilde{F}_{4}-\gamma^{i j} \tilde{F}_{3}^{i} \wedge \mathcal{A}_{1}^{j}+\frac{1}{2} \gamma^{i k} \gamma^{j \ell} \tilde{F}_{2}^{i j} \wedge \mathcal{A}_{1}^{k} \wedge \mathcal{A}_{1}^{\ell}-\frac{1}{6} \gamma^{i \ell} \gamma^{j m} \gamma^{k n} \tilde{F}_{1}^{i j k} \wedge \mathcal{A}_{1}^{\ell} \wedge \mathcal{A}_{1}^{m} \wedge \mathcal{A}_{1}^{n} \\
F_{3}^{i} & =\gamma^{j i} \tilde{F}_{3}^{j}+\gamma^{j i} \gamma^{k \ell} \tilde{F}_{2}^{j k} \wedge \mathcal{A}_{1}^{\ell}+\frac{1}{2} \gamma^{j i} \gamma^{k m} \gamma^{\ell n} \tilde{F}_{1}^{j k \ell} \wedge \mathcal{A}_{1}^{m} \wedge \mathcal{A}_{1}^{n} \\
F_{2}^{i j} & =\gamma^{k i} \gamma^{\ell j} \tilde{F}_{2}^{k \ell}-\gamma^{k i} \gamma^{\ell j} \gamma^{m n} \tilde{F}_{1}^{k \ell m} \wedge \mathcal{A}_{1}^{n} \\
F_{1}^{i j k} & =\gamma^{\ell i} \gamma^{m j} \gamma^{n k} \tilde{F}_{1}^{\ell m n} \\
\mathcal{F}_{2}^{i} & =\tilde{\mathcal{F}}_{2}^{i}-\gamma^{j k} \tilde{\mathcal{F}}_{1}^{i j} \wedge \mathcal{A}_{1}^{k} \\
\mathcal{F}_{1}^{i j} & =\gamma^{k j} \tilde{\mathcal{F}}_{1}^{i k}
\end{aligned}
$$

where the tilded quantities represent the unmodified pure exterior derivatives of the corresponding potentials, and $\gamma^{i j}$ is defined by

$$
\gamma^{i j}=\left[\left(1+\mathcal{A}_{0}\right)^{-1}\right]^{i j}=\delta^{i j}-\mathcal{A}_{0}^{i j}+\mathcal{A}_{0}^{i k} \mathcal{A}_{0}^{k j}+\cdots .
$$

Recalling that $\mathcal{A}_{0}^{i j}$ is defined only for $j>i$ (and vanishes if $j \leq i$ ), we see that the series terminates after a finite number of terms. The term $\mathcal{L}_{F F A}$ in (A.1) is the dimensional reduction of the $\tilde{F}_{4} \wedge \tilde{F}_{4} \wedge A_{3}$ term in $D=11$, and is given in lower dimensions in 16].

\section{References}

[1] J. Scherk and J.H. Schwarz, How to get masses from extra dimensions, Nucl. Phys. B153 (1979) 61.

[2] E. Bergshoeff, M. de Roo, M.B. Green, G. Papadopoulos and P.K. Townsend, Duality of type II 7-branes and 8-branes, Nucl. Phys. B470 (1996) 113: hep-th/9601150.

[3] P.M. Cowdall, H. Lü, C.N. Pope, K.S. Stelle and P.K. Townsend, Domain walls in massive supergravities, Nucl, Phys, B486 (1997) 49: hep-th/9608173.

[4] H. Lü and C.N. Pope, Domain walls from M-branes, Phys. Lett. B391 (1997) 39: hep-th/9611079.

[5] L.V. Lavrinenko, H. Lü and C.N. Pope, From topology to generalised dimensional reduction, Nucl. Phys. B492 (1997) 278: hep-th/9611134.

[6] H. Lü, C.N. Pope, T.A. Tran and K.-W. Xu, Classification of p-branes, NUTs, waves and intersections, hep-th/9708055.

[7] E. Cremmer and B. Julia, The $N=8$ supergravity theory-I-The Lagrangian, Phys. Lett. B80 (1978) 48; The SO(8) supergravity, Nucl. Phys. B156 (1979) 141. 
[8] B. Julia, Group disintegrations; E. Cremmer, Supergravities in 5 dimensions, in Superspace and Supergravity, Eds. S.W. Hawking and M. Rocek (Cambridge Univ. Press, 1981) $331 ; 267$.

[9] J. Maharana and J.H. Schwarz, Noncompact symmetries in string theory, Nucl. Phys. B390 (1993) 3: hep-th/9207016.

[10] E. Cremmer, B. Julia, H. Länd C.N. Pope, Dualisation of dualities. I, hep-th/9710119

[11] E. Cremmer, H. Lü, C.N. Pope and K.S. Stelle, Spectrum-generating symmetries for BPS solitons, hep-th/9707207.

[12] L. Castellani, R. D'Auria and P. Fré, Supergravity and superstrings, a geometric perspective. Vols 1-3, World Scientific, Singapore (1991).

[13] P.S. Howe, N.D. Lambert, P.C. West, A new massive type IIA supergravity from compactification, hep-th/9707139.

[14] L.J. Romans, Massive $N=2 a$ supergravities in ten dimensions, Phys. Lett. B169 (1986) 374 .

[15] J. Polchinski and E. Witten, Evidence for heterotic type I string duality, Nucl. Phys. B460 (1996) 525: hep-th/9510169.

[16] H. Lü and C.N. Pope, p-brane solitons in maximal supergravities, Nucl. Phys. B465 (1996) 127: hep-th/9512012.

[17] See, for example, M.P. Ryan and L.C. Shepley, Homogeneous Relativistic Cosmologies, (Princeton University Press, 1975).

[18] H. Lü and C.N. Pope, Multi-scalar p-brane solitons, Int. J. Mod. Phys. A12 (1997) 437: hep-th/9512153.

[19] H. Lü, C.N. Pope, E. Sezgin and K.S. Stelle, Stainless super p-branes, Nucl. Phys. B456 (1995) 669: hep-th/9508042.

[20] J.H. Schwarz, An $S L(2, \mathbb{Z})$ multiplet of type IIB superstrings, Phys. Lett. B360 (1995) 13; Erratum-ibid. B364 (1995) 252: hep-th/9508143.

[21] H. Lü, C.N. Pope, E. Sezgin and K.S. Stelle, Dilatonic p-brane solitons, Phys. Lett. B371 (1996) 46: hep-th/9511203. 
[22] P.S. Howe, Weyl superspace, hep-th/9707184. 